\title{
DNA Immunization Using in vivo Electroporation for Generating Monoclonal Antibodies Against Mouse IL-9R \\ Shogo Takatsuka ${ }^{1, \$, *}$, Hiroyuki Yamada ${ }^{1, \#}$, Kei Haniuda ${ }^{1}, \#$, Marina Ichihashi' ${ }^{11,}$ Joe Chiba $^{2}$ and Daisuke Kitamura ${ }^{1}$
}

\begin{abstract}
1Division of Molecular Biology, Research Institute for Biomedical Sciences (RIBS), Tokyo University of Science, Noda, Japan; ${ }^{2}$ Department of Biological Science and Technology, Tokyo University of Science, Tokyo, Japan; ${ }^{\$}$ Current address: Department of Chemotherapy and Mycoses, National Institute of Infectious Diseases, Tokyo, Japan

*For correspondence: tktk@niid.go.jp

\#Contributed equally to this work
\end{abstract}

[Abstract] Membrane proteins such as cytokine receptors and $G$ protein-coupled receptors can be drug targets. Recently, we have generated specific monoclonal antibodies (mAbs) against the mouse IL-9 receptor (IL-9R) and found that IL-9R on memory B cells have critical roles in T-dependent immune response. So far, most antibodies against cell surface proteins have been generated by immunization of animals with recombinant proteins produced in Escherichia coli (E. coli) or peptides derived from the protein. However, such antibodies often fail to recognize native proteins on cell surfaces because these antigens lack posttranslational modification and natural protein conformations. To circumvent such problems, we have developed a mouse immunization method, the DNA-immunization utilizing hyaluronidase and E. coli GroEL. Herein, we report an application of the original mouse immunization method in rats to generate anti-mouse IL-9R mAbs which could react with the native form of mouse IL-9R on cell surfaces. Thus, we suggest that the DNA-immunization method is feasible for generating monoclonal antibodies against cell surface proteins in rats.

Keywords: IL-9 receptor, Monoclonal antibodies, In vivo electroporation, GroEL, Hyaluronidase

[Background] Membrane proteins such as cytokine receptors and $G$ protein-coupled receptors have been regarded as medicinal drug targets, and generation of antibodies reactive with the native form of such membrane proteins would lead to antibody-drug development. However, most antibodies generated so far by immunization with a recombinant protein produced in Escherichia coli react only with the immunizing recombinant proteins, but not with the native proteins on cell surfaces. The same problems have often been experienced with immunization with peptides as antigens. In order to generate mAbs which could recognize the native proteins, we have developed the original DNA-immunization method in which plasmid DNA is directly transferred into mouse skeletal muscle in vivo utilizing Escherichia coli (E. coli) GroEL (Fujimoto et al., 2009; Takatsuka et al., 2011) and hyaluronidase. E. coli GroEL, is a molecular chaperone that is responsible for the transportation and refolding of proteins and GroEL fusion proteins are highly expressed in the soluble fraction (Furutani et al., 2005). Further, E. coli GroEL also acts as an adjuvant via TLR4 (Fujimoto et al., 2012). 
Pre-treatment with hyaluronidase enhances gene expression by prevention of tissue damage (McMahon et al., 2001). Furthermore, low molecular weight hyaluronan produced by hyaluronidase in vivo provides adjuvant effects via TLR2 and TLR4 (Scheibner et al., 2006, Gariboldi et al., 2008). The striated muscle is an excellent tissue for taking up and expressing genes that are transferred in the form of plasmid DNA (Wolff et al., 1990 and 1992, Davis et al., 1993; Ulmer et al., 1993; Aihara and Miyazaki, 1998). The longevity of myofibers allows the stable expression of transfected exogenous genes. Once induced, the expression of the transduced gene can last for at least 70 days thereafter (Wolff et al., 1990 and 1992; Davis et al., 1993; Ulmer et al., 1993; Yamazaki et al., 2011) and the expressed antigens are continuously exposed to the host immune system.

In the present paper, we report an application of the mouse DNA-immunization method in rats in order to generate anti-mouse IL-9R mAbs. IL-9 receptor (IL-9R) is composed of the IL-9Ra and common y chains and is expressed on mast cells, basophils, ILC2 cells and others (Renauld et al., 1992, Bauer et al., 1998; Noelle and Nowak, 2010; Wilhelm et al., 2011; Licona-Limón et al., 2013). IL-9 is produced by various immune cells, such as activated $T$ cells (exemplified by $T_{H} 9$ cells), mast cells, eosinophils, and type 2 innate lymphoid cells (ILC2 cells), and induces various functions depending on the target-cell types expressing its receptor (IL-9R) (Gounni et al., 2000; Stassen et al., 2000; Noelle and Nowak, 2010; Wilhelm et al., 2011; Licona-Limón et al., 2013). Thus, IL-9/IL-9R signaling is mainly involved in immune responses such as allergic inflammations. We successfully generated four rat-mouse hybridomas producing mAbs that react with the native form of mouse IL-9R on cell surfaces. Using these mAbs, we found that IL-9R is selectively expressed on memory B cells among B-lineage cells in the T-cell-dependent immune response. We also found that IL-9R is induced on B cells by stimulation through CD40, but suppressed by IL-4 or IL-21, which may explain the minimum expression of IL-9R on germinal center B cells (Takatsuka et al., 2018). Thus, rat mAbs generated by the DNA-immunization method turned out to be quite reliable tools in staining cell surface proteins, and this method can be extended to immunization for various cell surface antigens against which specific mAbs are yet to be obtained.

\section{Materials and Reagents}

1. Absorbent cotton

2. $1 \mathrm{ml}$ syringes (Terumo, catalog number: SS-01T)

3. $26 \mathrm{G}$ needles (Terumo, catalog number: NN-2613S)

4. Micro slide glass (Matsunami, catalog number: S-2226)

5. Cell strainer (Thermo, catalog number: 08-771-2)

6. Cell lines

a. B300-19 cells

IL-9R/B300-19 and EV/B300-19 cells: B300-19 cells (a mouse pre-B cell line), transduced with a retroviral vector containing II9r cDNA (pMXs-IL9R-IRES-hNGFR) or an empty vector (EV; pMXs-IRES-hNGFR). The B300-19 cell line was provided by Dr. Takashi Nakayama. 
The retroviral vectors and B300-19 transfectants generated by us will be available upon request.

b. Plat-E cells

The Plat-E cell line and an original pMXs vector were provided by Dr. Toshio Kitamura.

7. Lew/SSN rats (Female Lew/SSN rats were purchased from Sankyo Lab Service. Rats were immunized at 4 weeks of age)

8. Depilatory cream (general commercialized product)

9. Sodium pentobarbital (Kyoritsu Seiyaku, Somnopentyl Injection)

10. QIAGEN Plasmid Giga Kit (QIAGEN, catalog number: 12191)

11. Fugene HD (Promega, catalog number: E2311)

12. FITC-conjugated secondary antibodies [FITC-conjugated goat anti-rat $\lg G(\mathrm{H}+\mathrm{L})$ ] (SouthernBiotech, catalog number: 3050 )

13. FITC-anti-IgG1 (BD Bioscience, catalog number: 562580 )

14. PE-anti-CD138 (Biolegend, catalog number: 142503)

15. PE-anti-lgM (Thermo, catalog number: 12-5790-81)

16. PE-anti-IgD (Biolegend, catalog number: 405705)

17. PerCP/Cy5.5-anti-T and -B Cell activation antigen clone GL-7 (Biolegend, catalog number: 144609)

18. PE/Cy7-anti-CD38 (Thermo, catalog number: 25-0381-80)

19. APC/Cy7-anti-B220 (Biolegend, catalog number: 103223)

20. Biotin-NP $14-\mathrm{BSA}$ (in house)

21. Brilliant Violet 421 Streptavidin (Biolegend, catalog number: 405226)

22. MEM medium (Thermo, catalog number: 11095080)

23. Hyaluronidase (Sigma, catalog number: H3631-30KU)

24. $70 \%(v / v)$ Ethanol

25. Phosphate buffered saline $(\mathrm{pH} 7.4)$

26. Tris

27. EDTA

28. Sodium azide

29. Hyaluronidase solution (see Recipes)

30. Tris-EDTA buffer (see Recipes)

31. FACS Buffer (see Recipes)

32. Plasmid DNA solution (see Recipes)

\section{Equipment}

1. Electroporator (BTX, Electro Square Porator ECM830, Figure 1A)

2. Copper cables with IC hook (Toyoshima, made-to-order or general commercialized product, length: more than one meter, Figure 1A-1B) 
3. $26 \mathrm{G}$ two-stage needle (Toyoshima, made-to-order, Figure 1C-1D)

4. Gamma irradiation device (Atomic Energy of Canada Limited, Gammacell 40)

5. BD FACS Calibur

6. BD FACS Cantoll

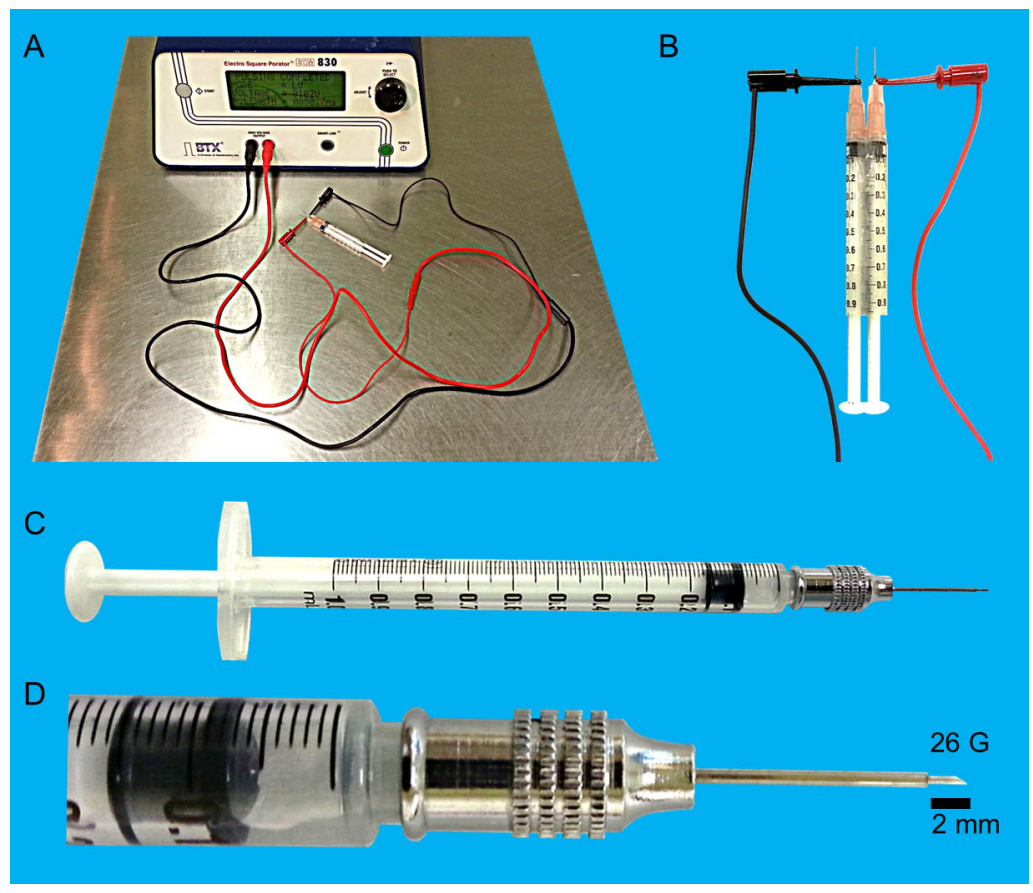

Figure 1. Details of the experimental equipment for DNA immunization. A. Electroporator.

B. Electrodes and copper cables with IC hook. C. $1 \mathrm{ml}$ syringe with a $26 \mathrm{G}$ two-stage needle. D. $26 \mathrm{G}$ two-stage needle.

\section{Software}

1. FlowJo (Tree Star, https://www.flowjo.com)

\section{Procedure}

1. Prepare and set up the electroporator (Figure 1A):

Voltage: $100 \mathrm{~V}$

Pulse length: $50 \mathrm{~ms}$

Pulse number: 6 ( $1 \mathrm{~s}$ interval)

Periodicity: $1 \mathrm{kHz}$.

2. Anesthetize rat with $24-36 \mathrm{mg} / \mathrm{kg}$ sodium pentobarbital. (Duration time: $30-60 \mathrm{~min}$ )

3. Depilate hindlimbs with depilatory cream (cream should stay for about $3 \mathrm{~min}$ ) (Figure 2A).

4. Sterilize hindlimbs using absorbent cotton impregnated with $70 \%(\mathrm{v} / \mathrm{v})$ ethanol. 
5. Inject $50 \mu \mathrm{l}$ of hyaluronidase solution into the quadriceps muscle using a $1 \mathrm{ml}$ syringe with a 26 G two-stage needle (Figure 2B).

6. Leave to stand for $10 \mathrm{~min}$.

7. Stab electrodes into the quadriceps muscle (Figures 1B and $2 \mathrm{C}$ ).

8. Inject $30 \mu \mathrm{l}$ of plasmid DNA solution into the same place where hyaluronidase solution pretreatment was done (Figure 2D).

9. Pulse by electroporator ( $100 \mathrm{~V}, 50 \mathrm{~ms}$ pulse length, $1 \mathrm{kHz}, 6$ times with each $1 \mathrm{~s}$ interval).

10. Repeat the same operation (Steps 3-9) to the other hindlimb (Figure 2A-2D). Note: The required time of operation (Steps 3-10) is about 20 min.
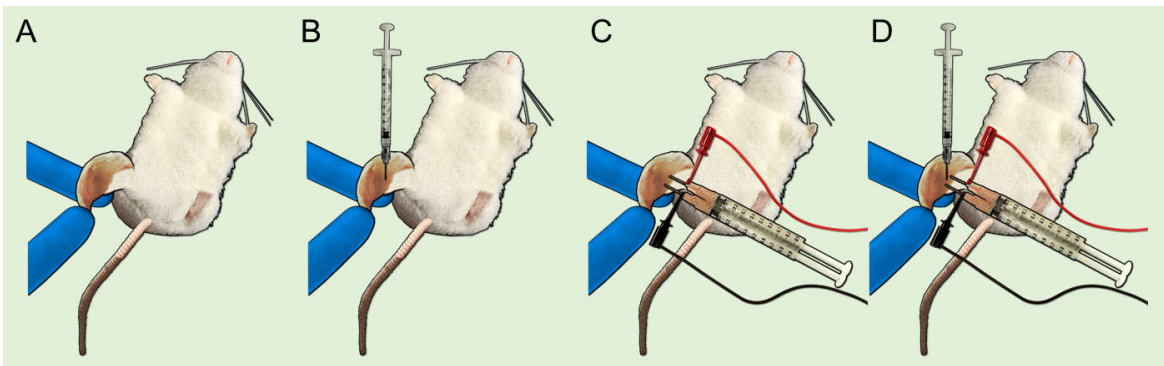

Figure 2. Experimental procedure of DNA immunization using in vivo electroporation. A.

Depilate hindlimbs. B. Inject hyaluronidase solution into quadriceps muscle and leave to stand for $10 \mathrm{~min}$. C. Stab the electrodes into the quadriceps muscle. D. Inject plasmid DNA solution into the same place where hyaluronidase solution pretreatment was done. Subsequently, pulse by electroporator. Repeat the same operation (A-D) to the other hindlimb.

11. Repeat the same DNA-immunization protocol 1, 3, 4, 7 and 9 weeks after the primary immunization, respectively (Figure 3).

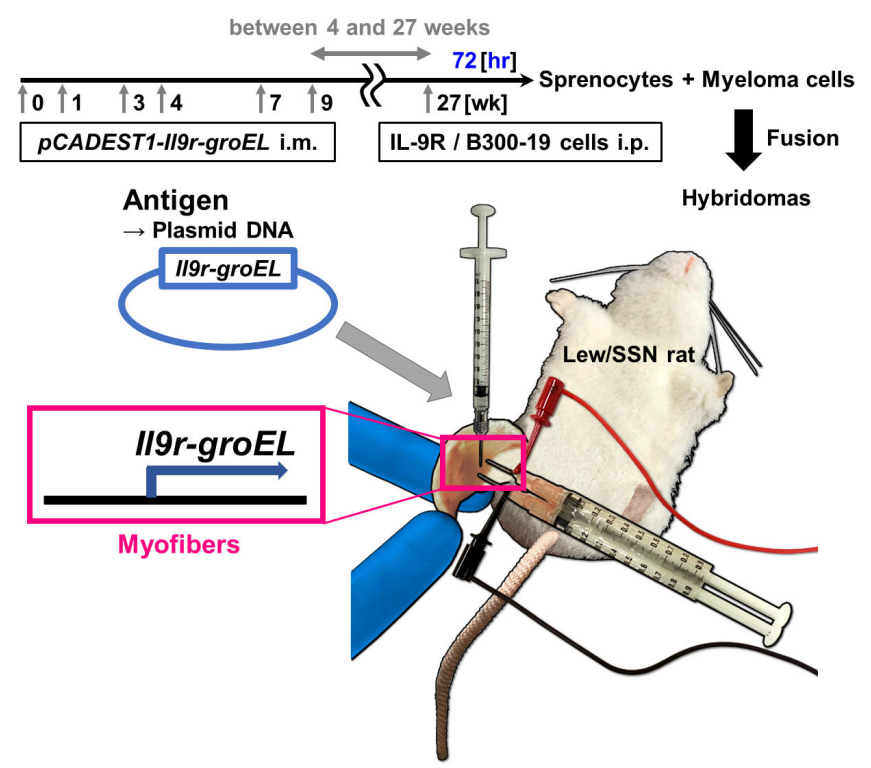

Figure 3. Overview of DNA immunization using rats. Scheme of DNA immunization using in 
vivo electroporation.

12. Irradiate (30 Gy) $1 \times 10^{9} \mathrm{IL}-9 \mathrm{R} / \mathrm{B} 300-19$ cells in PBS (preferably on ice) using Gamma irradiation device.

13. Transfer $1 \times 10^{9}$ irradiated IL-9R/B300-19 cells intraperitoneally 4-27 weeks after the final immunization.

14. Six or eleven weeks after the primary immunization, check sera of the immunized rats for anti-IL-9R IgG by flow cytometry using IL-9R/B300-19 cells. Detection with sera diluted by $1: 10^{4}$ or more is desirable (Figure $4 \mathrm{~A}$ ).

15. In order to generate hybridomas that produce monoclonal antibodies, collect splenocytes from the immunized rats 3 days after the cell transfer, and fuse them with mouse myeloma cell line (e.g., SP2/0) by a conventional method (e.g., Köhler and Milstein, 1975).

16. Screen the hybridomas for secretion of IgG Ab that stains IL-9R/B300-19 cells (and does not stain EV/B300-19 cells) using flow cytometry (Figure 4B).

A

Antisera_6 wk (1/100 dil)

Antisera_6 wk (1/1000 dil)

Antisera_6 wk (1/10000 dil)

Without Antisera

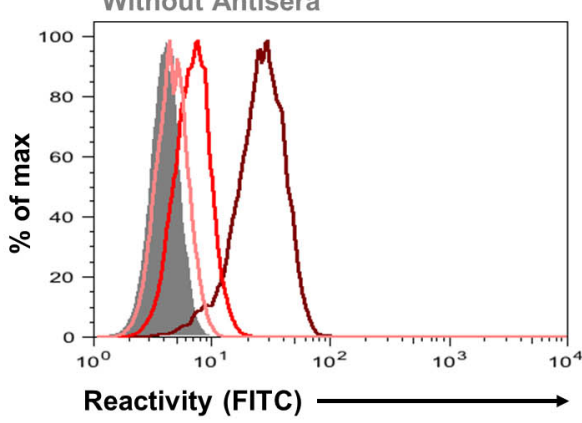

B

IL-9R/B300-19

EV/B300-19

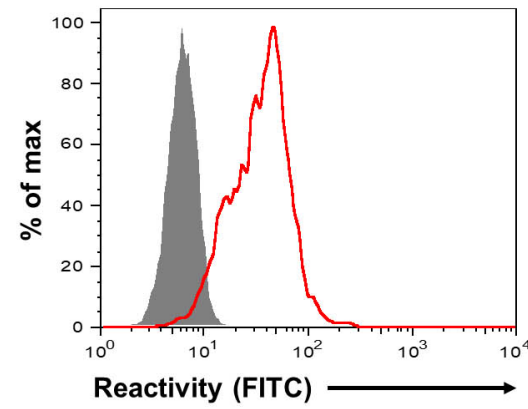

Figure 4. Titration of antisera and hybridoma screening. A. Titration of anti-IL9R antisera using flow cytometry. IL-9R/B300-19 cells were stained with sera, diluted (dil) as indicated, of a rat, immunized 6 weeks previously. B. An example of flow cytometric analysis in hybridoma screening. IL-9R/B300-19 or EV/B300-19 cells were stained with hybridoma supernatant. (A-B) Bound IgGs were revealed with FITC-conjugated anti-rat IgG Ab.

17. Stain the cells expressing IL-9R utilizing anti-mouse IL-9R (e.g., Figure 5) (Takatsuka et al., 2018). 


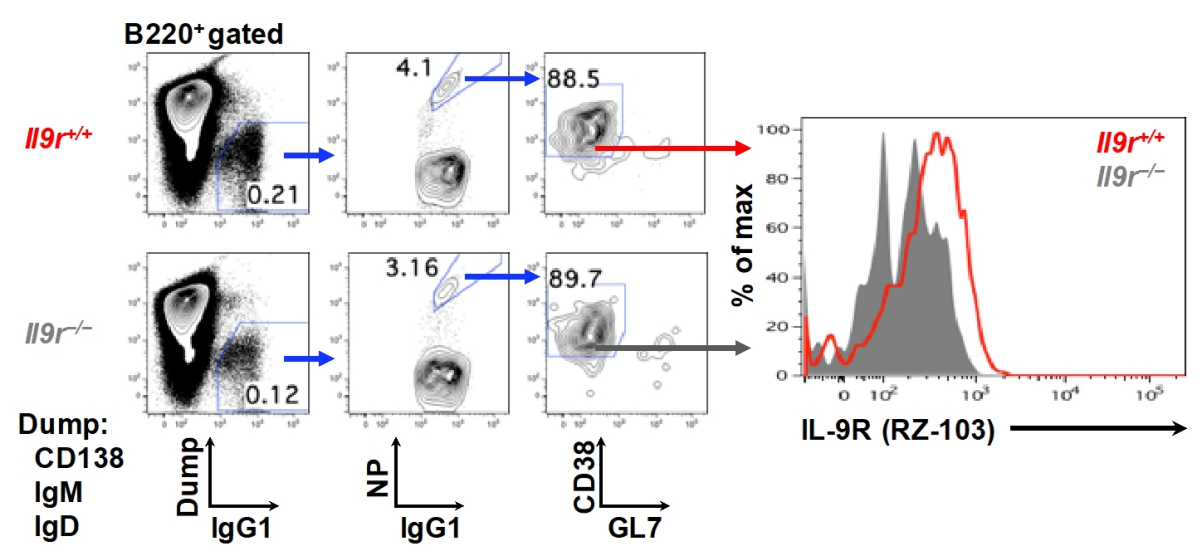

Figure 5. Detection of IL-9R expression on memory B cells. Flow cytometry analysis of IL-9R expression using anti-IL-9R mAb, RZ-103, (right panel) on NP-specific IgG1+ memory B cells in the spleen (gating strategies are shown on the left) of $119 r^{+/+}$or $119 r^{-1-}$ mice immunized i.p. with a hapten NP conjugated with chicken gamma globulin (NP-CGG) in alum 16 weeks earlier.

\section{Data analysis}

Flow cytometry analysis: For analysis, collect $>5,000$ events (Figure 4) or $>5,000,000$ events (Figure 5) for each sample. Exclude dead cells and cellular debris from analysis. Define singlet cells in a lymphocyte gate by forward, and side scatters. These cells can be visualized by the fluorescently labeled antibodies. (e.g., Figure 5: the analysis of the Flow Cytometry assay is done via FlowJo.)

\section{$\underline{\text { Notes }}$}

1. Retroviral transduction

a. Transfect pMXs-IL9R-IRES-hNGFR vectors into Plat-E cells by Fugene HD.

b. Two days after transfection, harvest the virus-containing supernatant and add to B300-19 cells.

2. Preparation of serum

a. Cut up rat's tail using a surgical knife and collect the peripheral blood $(50-100 \mu \mathrm{l})$.

b. Leave to stand the peripheral blood at $4{ }^{\circ} \mathrm{C}$, overnight.

c. Centrifuge the peripheral blood at $2,500 \times \mathrm{g}, 4^{\circ} \mathrm{C}$ for $10 \mathrm{~min}$.

d. Collect the serum $(20-40 \mu \mathrm{l})$ and store at $-20^{\circ} \mathrm{C}$.

3. Preparation of Single-cell suspensions from spleens

a. Grind the spleen by frosted glass portion of the slide glass in the MEM medium.

b. Filter splenocytes through a cell strainer.

4. Serum titer check using flow cytometry

a. Dilute the serum with FACS Buffer and add to $5 \times 10^{4} \mathrm{IL}-9 \mathrm{R} / \mathrm{B} 300-19$ cells.

b. Leave to stand on ice for $20 \mathrm{~min}$. 
c. Wash two times each with $200 \mu$ of FACS Buffer.

d. Add to FITC-conjugated secondary antibodies.

e. Leave to stand on ice for $20 \mathrm{~min}$.

f. Wash two times each with $200 \mu \mathrm{l}$ of FACS Buffer.

g. Go to flow cytometry analysis (see Data analysis).

5. Hybridoma screening using flow cytometry
a. Collect culture supernatants $(25-50 \mu \mathrm{l})$ of hybridomas and add to $5 \times 10^{4} \mathrm{IL}-9 \mathrm{R} / \mathrm{B} 300-19$ cells.
b. Leave to stand on ice for $20 \mathrm{~min}$.
c. Wash two times each with $200 \mu \mathrm{l}$ of FACS Buffer.
d. Add to FITC-conjugated secondary antibodies.
e. Leave to stand on ice for $20 \mathrm{~min}$.
f. Wash two times each with $200 \mu \mathrm{l}$ of FACS Buffer.
g. Go to flow cytometry analysis (see Data analysis).

\section{$\underline{\text { Recipes }}$}

1. Hyaluronidase solution

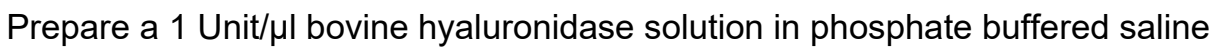

2. Tris-EDTA buffer

$10 \mathrm{mM}$ Tris, $\mathrm{pH} 7.5$

1 mM EDTA

3. FACS Buffer

PBS (-)

$0.5 \%$ BSA

2 mM EDTA

$0.05 \%$ sodium azide

4. Plasmid DNA solution

Prepare a $10 \mathrm{mg} / \mathrm{ml}$ DNA solution (in Tris-EDTA buffer) of a plasmid carrying a gene encoding IL-9R-GroEL fusion protein, driven by a CAG promoter, as previously described (Fujimoto et al., 2009)

\section{Acknowledgments}

We thank Y. Takatsuka for figure preparation; K. Sano for proofreading. This work was supported by the Japan Society for the Promotion of Science KAKENHI, Grant-in-Aid for Scientific Research (B) (16H05206) and Grant-in-Aid for Scientific Research on Innovative Areas (25111512). 


\section{Competing interests}

All authors declare that they have no competing interests.

\section{Ethics}

Lew/SSN rats were maintained in an animal facility in Research Institute for Biomedical Sciences, Tokyo University of Science under specific pathogen-free conditions and were treated under protocols approved by the Animal Care and Use Committee of the Tokyo University of Science.

\section{$\underline{\text { References }}$}

1. Aihara, H. and Miyazaki, J. (1998). Gene transfer into muscle by electroporation in vivo. Nat Biotechnol 16(9): 867-870.

2. Bauer, J. H., Liu, K. D., You, Y., Lai, S. Y. and Goldsmith, M. A. (1998). Heteromerization of the gammac chain with the interleukin-9 receptor alpha subunit leads to STAT activation and prevention of apoptosis. J Biol Chem 273(15): 9255-9260.

3. Davis, H. L., Whalen, R. G. and Demeneix, B. A. (1993). Direct gene transfer into skeletal muscle in vivo: factors affecting efficiency of transfer and stability of expression. Hum Gene Ther 4(2): 151-159.

4. Fujimoto, A., Kosaka, N., Hasegawa, H., Suzuki, H., Sugano, S. and Chiba, J. (2012). Enhancement of antibody responses to native $\mathrm{G}$ protein-coupled receptors using $E$. coli GroEL as a molecular adjuvant in DNA immunization. $J$ Immunol Methods 375(1-2): 243-251.

5. Fujimoto, A., Takatsuka, S., Ishida, I. and Chiba, J. (2009). Production of human antibodies to native cytokine receptors using the genetic immunization of KM mice. Hum Antibodies 18(3): 75-80.

6. Furutani, M., Hata, J., Shomura, Y., Itami, K., Yoshida, T., Izumoto, Y., Togi, A., Ideno, A., Yasunaga, T., Miki, K. and Maruyama, T. (2005). An engineered chaperonin caging a guest protein: Structural insights and potential as a protein expression tool. Protein Sci 14(2): 341-350.

7. Gariboldi, S., Palazzo, M., Zanobbio, L., Selleri, S., Sommariva, M., Sfondrini, L., Cavicchini, S., Balsari, A. and Rumio, C. (2008). Low molecular weight hyaluronic acid increases the self-defense of skin epithelium by induction of $\beta$-defensin 2 via TLR2 and TLR4. J Immunol 181(3): 2103-2110.

8. Gounni, A. S., Nutku, E., Koussih, L., Aris, F., Louahed, J., Levitt, R. C., Nicolaides, N. C. and Hamid, Q. (2000). IL-9 expression by human eosinophils: regulation by IL-1 $\beta$ and TNF- $\alpha$. $J$ Allergy Clin Immunol 106(3): 460-466.

9. Kohler, G. and Milstein, C. (2005). Continuous cultures of fused cells secreting antibody of predefined specificity. 1975. J Immunol 174(5): 2453-2455. 
10. Licona-Limon, P., Henao-Mejia, J., Temann, A. U., Gagliani, N., Licona-Limon, I., Ishigame, H., Hao, L., Herbert, D. R. and Flavell, R. A. (2013). Th9 cells drive host immunity against gastrointestinal worm infection. Immunity 39(4): 744-757.

11. McMahon, J. M., Signori, E., Wells, K. E., Fazio, V. M. and Wells, D. J. (2001). Optimisation of electrotransfer of plasmid into skeletal muscle by pretreatment with hyaluronidase-increased expression with reduced muscle damage. Gene Ther 8(16): 1264-1270.

12. Noelle, R. J. and Nowak, E. C. (2010). Cellular sources and immune functions of interleukin-9. Nat Rev Immunol 10(10): 683-687.

13. Renauld, J. C., Druez, C., Kermouni, A., Houssiau, F., Uyttenhove, C., Van Roost, E. and Van Snick, J. (1992). Expression cloning of the murine and human interleukin 9 receptor cDNAs. Proc Natl Acad Sci U S A 89(12): 5690-5694.

14. Scheibner, K. A., Lutz, M. A., Boodoo, S., Fenton, M. J., Powell, J. D. and Horton, M. R. (2006). Hyaluronan fragments act as an endogenous danger signal by engaging TLR2. J Immunol 177(2): 1272-1281.

15. Stassen, M., Arnold, M., Hultner, L., Muller, C., Neudorfl, C., Reineke, T. and Schmitt, E. (2000). Murine bone marrow-derived mast cells as potent producers of IL-9: costimulatory function of IL-10 and kit ligand in the presence of IL-1. J Immunol 164(11): 5549-5555.

16. Takatsuka, S., Sekiguchi, A., Tokunaga, M., Fujimoto, A. and Chiba, J. (2011). Generation of a panel of monoclonal antibodies against atypical chemokine receptor CCX-CKR by DNA immunization. J Pharmacol Toxicol Methods 63(3): 250-257.

17. Takatsuka, S., Yamada, H., Haniuda, K., Saruwatari, H., Ichihashi, M., Renauld, J. C. and Kitamura, D. (2018). IL-9 receptor signaling in memory B cells regulates humoral recall responses. Nat Immunol 19(9): 1025-1034.

18. Ulmer, J. B., Donnelly, J. J., Parker, S. E., Rhodes, G. H., Felgner, P. L., Dwarki, V. J., Gromkowski, S. H., Deck, R. R., DeWitt, C. M., Friedman, A. and et al. (1993). Heterologous protection against influenza by injection of DNA encoding a viral protein. Science 259(5102): 1745-1749.

19. Wilhelm, C., Hirota, K., Stieglitz, B., Van Snick, J., Tolaini, M., Lahl, K., Sparwasser, T., Helmby, H. and Stockinger, B. (2011). An IL-9 fate reporter demonstrates the induction of an innate IL-9 response in lung inflammation. Nat Immunol 12(11): 1071-1077.

20. Wolff, J. A., Ludtke, J. J., Acsadi, G., Williams, P. and Jani, A. (1992). Long-term persistence of plasmid DNA and foreign gene expression in mouse muscle. Hum Mol Genet 1(6): 363-369.

21. Wolff, J. A., Malone, R. W., Williams, P., Chong, W., Acsadi, G., Jani, A. and Felgner, P. L. (1990). Direct gene transfer into mouse muscle in vivo. Science 247(4949 Pt 1): 1465-1468.

22. Yamazaki, T., Nagashima, M., Ninomiya, D., Arai, Y., Teshima, Y., Fujimoto, A., Ainai, A., Hasegawa, H. and Chiba, J. (2011). Passive immune-prophylaxis against influenza virus infection by the expression of neutralizing anti-hemagglutinin monoclonal antibodies from plasmids. Jpn J Infect Dis 64(1): 40-49. 